\title{
A Method for Efficient and Minimal Utilization of Mobile Resources for Multimedia Based on Interactive Streaming
}

\author{
Muhsina Muhammed ${ }^{1}$, Hafsath C. A. ${ }^{2}$ \\ ${ }^{1,2}$ Dept of CSE, Ilahia College of Engineeering and Technology, Kerala, India
}

\begin{abstract}
The dramatic emergence and exponential growth of mobile devices have made them one of the fast growing consumer commodities in history and are now dominating the appliance market. The spectrum bears a wide range of devices like smartphones, notebooks, tablets, ultrabooks, laptops, etc providing umpteen services. As users demand services wherever they go and whenever they need, mobile devices rely on wireless access for connectivity to gather information and leverage services. Low processing power, limited RAM, intermittent, low bandwidth and high latency data connections and impact on battery life are the key mobile challenges and when it comes to mobile multimedia services these limitations manifold. This juncture made way for a myriad of research works in this field to provide the best services within these limitations. This paper discusses about how to provide efficient multimedia media services with regard to the limited mobile resources and dynamic bandwidth scenario. Here, a method is being proposed to tackle efficient and minimal usage of mobile resources through interactive streaming where network and device parameters are collected and analyzed to enhance the multimedia experience, and thereby using the collected data for multimedia transcoding thus avoiding any wastage of bandwidth and mobile resources.
\end{abstract}

Key words: Cloud Multimedia, Network and Device aware, Interactive streaming.

\section{Introduction}

Mobile devices are spreading faster than any other consumer technology in history. Smart phones and tablets have mushroomed sparking a new wave of demand for mobile services. Today's users not only expect ubiquitous network connections, but also seek a rich media experience wherever they go and whenever they need. This act of perseverance for mobile media gives rise to a multifold of technical challenges. It is hard to sustain satisfactory quality of experience over inherently unreliable wireless links, as mobile devices rely on wireless access to connect to the outside world to gather information and use services. The types of wireless networks used are varied, consisting of a multitude of technologies that have different characteristics [2] [3]. This variability results in different bandwidth capability, round trip time and reliability of the link. Wireless connections are also more prone to errors because of exposure to various sources of noise and diminished reliability due to limitations in coverage. All these factors affect the networked services, but particularly multimedia services such as video streaming that rely on high throughput and high reliability of the link for successful playback.

Large-scale, simultaneous delivery of media contents tends to swamp existing mobile network infrastructure. Moreover, a fundamental gap exists between the resource demands of computationally expensive multimedia applications and the capabilities afforded by battery-operated mobile devices.

Mobile Cloud Computing (MCC) has revolutionized the way in which mobile users across the globe leverage services. Mobile cloud computing encompass wider range of applications presented to mobile users away from conventional applications by supporting hardware, 3D virtual environments, and large storage capacity, also users share the cloud infrastructure to their friends. MCC integrates cloud computing into the mobile environment and overcomes obstacles related to performance (e.g. battery life, bandwidth, service delay and storage), environments (e.g. scalability, heterogeneity, availability) and security (e.g. reliability and privacy). Thanks to the increase of great video compression techniques such as H.264 and MPEG-4, it is currently possible to merge audio, video and data inside the same signal and transmit it over packet based wireless networks. This technology can offer these hardware resources cost-effectively.

After its debut in personal computers and home entertainment devices, streaming video has found its place in the mobile environment as well. However, the characteristics of the mobile devices present a different set of challenges for delivering satisfactory video services. They are typically smaller in size designed to be handheld or carried on person in some manner. They have reduced screen size and somewhat limited computational power due to restrictions in size and power consumption. These technical challenges necessitate the adaptation of the content to the particular device so that successful reproduction is possible and desired quality is met. When the mobile device is exposed to unadjusted content in spatial resolution and frame-rate, unnecessary penalties in computational load are incurred mostly due to processing of the downscaling. This in turn leads to higher power consumption and can lower the quality if the device fails to execute the task on time. 
In general the issue of over and under provisioning is present in any video streaming service [4]. However, in mobile streaming it is particularly evident due to specific limitations of the mobile devices and the larger variety in the design of the user interface and the mode of interaction.

Furthermore, due to the specific mechanics of consumption of the content in handheld devices the perception of quality is different than in desktop or large screen devices. This creates the need for separate exploration of the process of optimizing the tradeoff between the used resources and delivered quality from traditional video delivery systems. To satisfy the ambition to deliver high quality of the service to its customers, providers need foremost an accurate estimation of the perceived quality. In order to be accurate in this estimation, they have to account for the unique characteristics of the mobile devices. These characteristics range from the way that the device is held, and used, to its screen size and computational power as well as the user expectations.

In this paper, a method to tackle efficient and minimal usage of mobile resources for leveraging multimedia services through interactive streaming is proposed. Here the system deals with how to provide efficient multimedia service in the context of limited mobile resources and dynamic bandwidth. This is done as the network and device parameters are collected and analyzed to enhance the multimedia experience, and thereby using the collected data for multimedia transcoding thus avoiding any wastage of bandwidth and mobile resources. Network bandwidth is calculated using Exponentially Weighted Moving Average. The libfaac and MPEG-4 are two transcoding techniques used here for the simplicity and effectiveness. The system avails various multimedia services like streaming, downloading and uploading of multimedia files with more efficiency and speed. This system also maintains authentication and $\log$ mechanisms to improve its overall accordance. A Bayesian Theory is used to infer at what resolution and frame rate an entire multimedia file can be decoded completely and smoothly.

\section{Related Works}

Zhou and Wang [5], studies cloud resource scheduling where they consider the blind scenario, with unknown system parameters such as user demand rate and server service time. By formulating the resource scheduling problem as a finite horizon probability-constrained stochastic optimization, they develop a blind scheduling algorithm that performs well in terms of fairness, simplicity, and asymptotic optimality. Lai et al. [1] present a candidate solution where a dynamic network estimation module attempts to forecast future bandwidth changes based on past observations. A network and device-aware Bayesian prediction module infers the appropriate video format from features and available resources of mobile devices. A prototype of the proposed system realizes real-time transcoding operations within the map-reduce framework in cloud computing. A different approach is investigated by $\mathrm{Zhu}, \mathrm{Li}$, and Chen [6] in which they present a video delivery framework that integrates scalable video coding (SVC) with multi-path routing. Their design leverages the storage and computing power of the cloud to collect network status and calculate video streaming routes in a centralized manner. Several multipath provisioning algorithms are evaluated for cloud-assisted SVC streaming to heterogeneous clients. Sardis et al. [7] in their work has raised the concern that the Internet may not respond fast enough to real-time services. They envision an alternative solution: serving individual geographical regions from small, local clouds. Such a design relieves the Internet from backbone congestion. Meanwhile, users can still access services from other regions, if needed.

Chen et al [8] aim at improving mobile video streaming with cloud computing. Their solution deploys video agents in the cloud on behalf of individual mobile users, and elastically allocates cloud resources for accelerating video encoding and delivery processes. Knowledge of users' social relationships is used to improve content pre-fetching efficiency. In their work CloudMoV, Wu et al. [9] utilizes the cloud to emulate the livingroom video watching experience for a group of disparate mobile users. The proposed platform combines several elements: flexible video transcoding, maximizing battery efficiency of mobile devices, and supporting spontaneous social interactions. CloudMoV is implemented on Amazon EC2 and Google App Engine. Its performance has been evaluated in real-world experiments. The growing amount of images and videos captured by smart phones has increased the appeal of automatic or semiautomatic management of personal media contents. Large scale image annotation is essential in fulfilling this task. Tao et al. [10] study cloud-based image annotation in and their proposed scheme compresses images using Hamming compressed sensing and conducts semantic annotation through a novel Hessian regularized support vector machine in the cloud. Evaluations of this approach yield promising results on the PASCAL VOC07 dataset. Xue et al. [11] exploits the availability of highly correlated contents in the cloud for image coding in their work. They present an image coding scheme that generates a baseline from a down-sampled version of the original image, and encodes the scale-invariant feature transform (SIFT) vectors in a predictive manner. The paper reports significantly higher compression ratios over conventional image coding, such as JPEG, JPEG2000, and intra-coding in HEVC, for data sets containing highly correlating images. 
Bisio et al. [12] propose a new Location Recognition algorithm for Automatic Check-In applications (LRACI). LRACI is designed for scenarios where traditional approaches, such as Global and Hybrid Positioning Systems (GPS/HPS), tend to fail. It opportunistically exploits the presence of Wi-Fi access points to complement existing GPS/HPS signals. The design hinges on two key ideas: sliding-window based filtering of GPS/HPS signals and a novel method for calculating Wi-Fi fingerprints. Its Android-based implementation can achieve a location recognition accuracy of $90 \%$. Wang and Dey [13] combines an extensive overview of cloudbased mobile media with original technical contributions. In this paper, they sketch out early marketing trends and three major challenges: guaranteeing user experience, managing communication and computation costs, and scaling services to millions of users. Using cloud mobile gaming as a concrete application example, the authors demonstrate the effectiveness of a novel joint rendering and encoder bit rate adaptation (JREA) technique in addressing all three challenges. The paper continues to discuss promising future research directions for fully reaping the benefits of cloud computing in mobile media networks.

\section{Proposed System}

In this system, to access a multimedia file from a cloud server a mobile device user needs to register its information (First Name, Last Name, Mail id) with the server. Once registered, the mobile device can login to the server and avails the various services provided. Firstly, the server will retrieve the mobile device and network parameters, based on which a profile is created for the mobile device which will be stored in the server database. The server can use this information to select the best format for carrying out various multimedia services like streaming, downloading and uploading. The system wards off any wastage of network or device resources by libfaac transcoding technique. FAAC stands for Freeware Advanced Audio Coder. The FAAC encoder is an audio compression computer program that creates AAC (MPEG-2 AAC/MPEG-4 AAC) sound files from other formats (usually, CD-DA audio files). It contains a library (libfaac) that can be used by other programs. A user log is provided in the project for keeping track of the user activities.

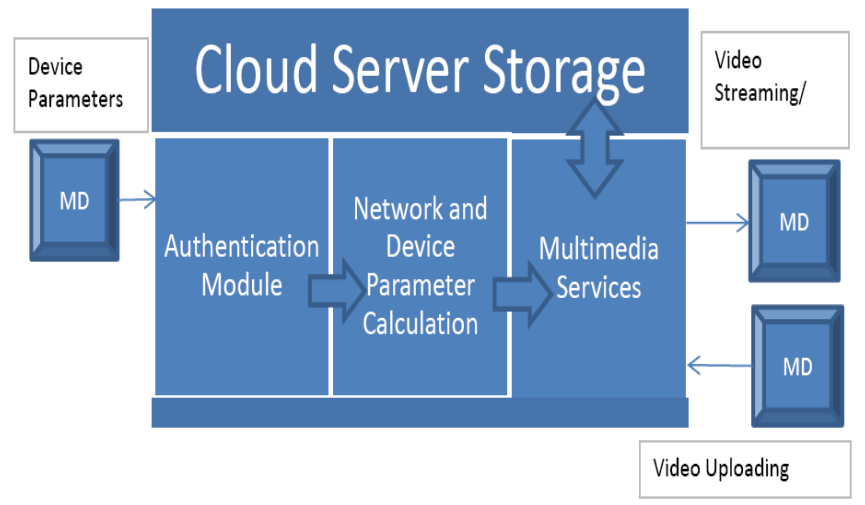

Figure1. Proposed System Architecture

\section{Solution Methodology}

The proposed system has following modules that are described below,

- Authentication

- Network and device parameters calculation

- Interactive communication

- Video download/streaming

- Video upload

\section{Authentication}

The mobile devices are authenticated by providing a signin/signup module in the system with which any new user can register into the server. A new mobile device user accessing the cloud server should sign up by providing information like username, password, and email id and henceforth can sign in using the username and password. Apart from ensuring the authentication of the mobile devices, this module enables the server to keep track of the online activities of the device.

\section{Network and Device Parameter Calculation}

This module calculates network and device parameters like bandwidth, network provider and type of network, OS version, SIM state and device type., based on which it creates a user profile for the mobile device and store it in the server database. As the bandwidth tends to change from time to time it should be calculated 
with care and precision. It is done mainly based on the measurement-based prediction concept; however, it further develops the Exponentially Weighted Moving Average (EWMA). The EWMA uses the weights of the historical data and the current observed value to calculate gentle and flexible network bandwidth data for the dynamic adjustment of weights. All these parameters are analyzed and based on which the system estimates whether the current network state is suitable for a mobile device for entertaining a given multimedia access.

Also it is necessary to determine an appropriate video format according to the available resources of various devices and the changing network conditions. A Bayesian Theory is used to infer at what resolution and frame rate an entire multimedia file can be decoded completely and smoothly. As for the three video parameters of frame rate, resolution and bit rate, the bit rate depends on the frame rate and resolution, so the Bayesian network adopts the frame rate and resolution as the video input features and uses battery power, power consumption and computing power as device features based on which the inference that completed playback and in-time decoding is met by a selected video format is reached.

\section{Interactive Communication}

This module adaptively adjusts the streaming flow with various transcoding techniques. Video transcoding performs one or more operations, such as bit rate and format conversions, to transform one compressed video stream to another [14]. Transcoding can enable multimedia devices of diverse capabilities and formats to exchange video content on heterogeneous network platforms such as the Internet. This project uses transcoding techniques like FAAC and MPEG-4.

FAAC stands for Freeware Advanced Audio Coder. The FAAC encoder is an audio compression computer program that creates AAC (MPEG-2 AAC/MPEG-4 AAC) sound files from other formats (usually, CD-DA audio files). It contains a library (libfaac) that can be used by other programs. AAC files are commonly used in computer programs and portable music players, being Apple Inc.'s recommended format for the company's iPod music player.

Some of the features that FAAC has are: cross-platform support, "reasonably" fast encoding, support for more than one "object type" of the AAC format, multi-channel encoding, and support for Digital Radio Mondiale streams. It also supports multi-channel streams, like 5.1. The MPEG-4 object types of the AAC format supported by FAAC are the "Low Complexity" (LC), "Main", and "Long Term Prediction" (LTP). The MPEG-2 AAC profiles supported by FAAC are LC and Main. The SBR and PS object types are not supported, so the HE-AAC and HE-AACv2 profiles are also not supported. The object type "Low Complexity" is the default and also happens to be used in videos meant to be playable for portable players (like Apple's iPod) and used by video-hosting sites (like YouTube).

MPEG-4 has standardized a scalable coding scheme referred to as fine granularity scalability (FGS). With this scheme, a base layer and an enhancement layer bit streams are generated. The base is coded with the usual motion-compensated DCT techniques and is compliant to the Advanced Streaming Profile, while the enhancement is coded with a bit-plane coding method that is supported in the FGS Profile. The key advantage to this coding method is that it allows the enhancement layer bit stream to be truncated into any number of bits within each frame to provide partial enhancement proportional to the number of bits decoded for each frame. The quality of the reconstructed frame is proportional to the number of enhancement bits received.

\section{Video Download/Streaming}

After the user gains access to the application by providing the username and password, the home screen will display video download and upload options. User can select the 'video download' option which redirects to a page for calculating networks and device parameters. Here, clicking the 'Get Mobile Info' button will retrieve and display the values of these parameters. After successfully calculating the values, it redirects to a page listing all the available videos stored on the server. The user can select the desired file, upon selection a new screen appears asking the user whether to stream or download the file. By streaming user can view the video without coping it to the device while download option will download and save a copy of multimedia file in user's device. After the completion of the task user can go back to the previous menu of the application for availing further services.

\section{Video Upload}

Gaining access to the application enables the user to leverage two different services like video downloading and video uploading. On selecting the 'upload video' option from the home page of the application, the user is redirected to a web page where he can select the multimedia file for uploading from the device. Using the browse button on the web page user can select any file located in the device memory and then click the upload button to upload the file into the server. The file is stored at a dedicated folder in the server, and can be accessed by any other authentic user for downloading. 


\section{Conclusion And Future Works}

In contrast to the limitations in the field of mobile computing, it is quite interesting to watch the pace with which this wonderful field is evolving. As limitation being strength has sparkled interest in numerous researchers thereby paving way for many cutting edge technologies in this field. The mobile challenges that need a quick focus are the low bandwidth and high latency data connections and impact on battery life, peculiarly coming to mobile multimedia services as these shortcomings manifold. In this paper, a method to tackle efficient and minimal usage of mobile resources for leveraging multimedia services through interactive streaming is proposed. Here the system deals with how to provide efficient multimedia service in the context of limited mobile resources and dynamic bandwidth. This is done as the network and device parameters are collected and analyzed to enhance the multimedia experience, and thereby using the collected data for multimedia transcoding thus avoiding any wastage of bandwidth and mobile resources. The libfaac transcoding technique is used here for its simplicity and effectiveness. The system avails various multimedia services like streaming, downloading and uploading of multimedia files with more efficiency and speed. This system also maintains authentication and $\log$ mechanisms to improve its overall accordance. The project can be further enhanced by addressing other limitations of mobile computing like security standards and potential health hazards. Encompassing the vast and diverse arena of multimedia provides scope for future studies in the field.

\section{Acknowledgement}

The authors wish to thank the Management, Principal and Head of the department (CSE) of Ilahia College of Engineering and Technology for their support and help in completing this work.

\section{Reference}

[1]. Chin-Feng Lai, Honggang Wang, Han-Chieh Chao and Guofang Nan, “A Network and Device Aware QoS Approach for Cloud Mobile Streaming," IEEE Transactions on Multimedia, Vol. 15, No. 4, June 2013.

[2]. G. Zhang, Y. G. Wen, J. Zhu, and Q. H. Chen, "On delay minimization for file-based content uploading to media cloud via collaborative wireless networks (invited paper)," in Proc. 2011 Int. Conf. Wireless Commun. and Signal Process. (WCSP1), Nanjing, China, Nov. 9-11, 2011.

[3]. K. E. Psannis, Y. Ishibashi, and M.G.Hadjinicolaou, "QoS for wireless interactive multimedia streaming," in Proc. ACM Workshop QoS and Security for Wireless and Mobile Networks, 2007, pp. 168-171.

[4]. A. Garcia and H. Kalva, "Cloud transcoding for mobile video content delivery," in Proc. 2011 IEEE Int. Conf. Consumer Electronics (ICCE), 2011, pp. 379-380.

[5]. Liang Zhou and Haohong Wang, "Toward Blind Scheduling in Mobile Media Cloud: Fairness, Simplicity, and Asymptotic Optimality," IEEE Transactions on Multimedia, Vol. 15, Issue: 4, 2013, pp. 735-746.

[6]. Zuqing Zhu, Suoheng Li and Xiaoliang Chen, "Design QoS-Aware Multi-Path Provisioning Strategies for Efficient Cloud-Assisted SVC Video Streaming to Heterogeneous Clients," IEEE Transactions on Multimedia, Vol.15, Issue: 4, June 2013.

[7]. F. Sardis, G. Mapp, J. Loo and M. Aiash, "On the Investigation of Cloud-based Mobile Media Environments with ServicePopulating and QoS-aware Mechanisms,” IEEE Transactions on Multimedia, Vol. 15, Issue: 4, June 2013, pp. 769-777.

[8]. Xiaofei Wang, Min Chen, T.T. Kwon and J.T. Yang, "AMES-Cloud: A Framework of Adaptive Mobile Video Streaming and Efficient Social Video Sharing in the Clouds," IEEE Transactions on Multimedia, Vol. 15, Issue: 4, 2013, pp. 811-820.

[9]. Yu Wu, Zhizhong Zhang, C. Wu and Zongpeng Li, "CloudMoV: Cloud-based Mobile Social TV," IEEE Transactions on Multimedia, Vol. 15, Issue: 4, June 2013, pp. 821-832.

[10]. Dapeng Tao, Lianwen Jin, Weifeng Liu and Xuelong Li, "Hessian Regularized Support Vector Machines for Mobile Image Annotation on the Cloud," IEEE Transactions on Multimedia, Vol. 15, Issue: 4, June 2013.

[11]. Huanjing Yue, Xiaoyan Sun, Jingyu Yang and Feng Wu, "Cloud-based Image Coding for Mobile Devices — Toward Thousands to One Compression,” IEEE Transactions on Multimedia, Vol. 15, Issue: 4, June 2013, pp. 845-857.

[12]. Igor Bisio, Fabio Lavagetto, Mario Marchese and Andrea Sciarrone, "GPS/HPS- and Wi-Fi Fingerprint- based Location Recognition for Check-In Applications over Smartphones in Cloud-based LBSs," IEEE Transactions on Multimedia, Vol. 15, Issue: 4, 2013, pp. 858-869.

[13]. Shaoxuan Wang and Sujit Dey, "Adaptive Mobile Cloud Computing to Enable Rich Mobile Multimedia Applications," IEEE Transactions on Multimedia, Vol. 15, Issue: 4, June 2013.

[14]. Ishfaq Ahamd, Xiaohui Wei, Yu Sun and Ya-Qin Zhang, "Video Transcoding: An Overview of Various Techniques and Research Issues," IEEE Transactions on Multimedia, Vol. 7, No. 5, October 2005. 